\title{
Magnetic resonance imaging assessment of cardiac function in a swine model of hibernating myocardium 3 months following bypass surgery
}

Laura L. Hocum Stone, PhD, ${ }^{a}$ Cory Swingen, $\mathrm{PhD},{ }^{a}$ Christopher Holley, MD, ${ }^{\mathrm{a}}$ Christin Wright, BA, Erin Chappuis, ${ }^{a}$ Herbert B. Ward, MD, ${ }^{a}$ Edward O. McFalls, MD, PhD, ${ }^{b}$ and Rosemary F. Kelly, MD

\begin{abstract}
Objective: Clinical studies demonstrate delayed recovery of hibernating myocardium (HM) following coronary artery bypass graft (CABG) surgery. Cardiac magnetic resonance (CMR) imaging is effective in identifying HM in clinical settings. Our animal model of HM shows partial but incomplete functional recovery 1 month following CABG using echocardiography. This study uses CMR imaging to determine completeness of recovery 3 months post-CABG.
\end{abstract}

Methods: Swine $(\mathrm{N}=12)$ underwent left anterior descending artery (LAD) 1.5$\mathrm{cm}$ constrictor placement creating a territory of HM over 12 weeks. CMR at 12 weeks confirmed hibernation without infarction $(\mathrm{N}=12)$. Off-pump left internal thoracic artery (LITA) to the LAD was performed in 9 animals. Three animals were killed as HM controls. CMR imaging was repeated in revascularized animals before death at $1(\mathrm{n}=4)$ or 3 months $(\mathrm{n}=5)$. CMR imaging was performed at baseline and with dobutamine infusion $(5 \mu \mathrm{g} / \mathrm{kg} / \mathrm{min})$.

Results: Twelve weeks after constrictor placement, CMR imaging confirmed viability in $\mathrm{LAD}$ region and LAD stenosis in all animals. In HM, wall thickening is reduced at baseline but with contractile reserve present during dobutamine infusion. Following revascularization, CMR imaging confirmed patent LITA graft $(\mathrm{n}=9)$. Analysis of baseline regional function shows incomplete recovery of $\mathrm{HM}$ following $\mathrm{CABG}$, with reduced contractile reserve at both 1 and 3 months post-CABG.

Conclusions: CMR imaging provides accurate spatial resolution of regional contractile function and confirms the presence of HM at 12 weeks following instrumentation of the LAD. Three months following CABG, partial recovery of HM with contractile reserve is present in the single LAD territory. ( $\mathrm{J}$ Thorac Cardiovasc Surg 2017;153:582-90)

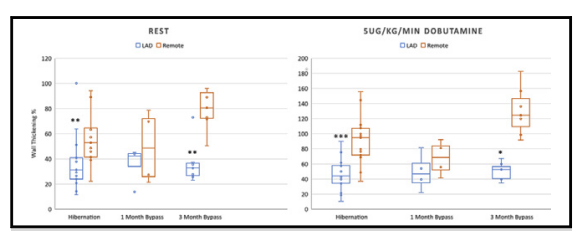

Cardiac magnetic resonance imaging demonstrates partial regional recovery of hibernating myocardium in swine 3 months following coronary artery bypass graft surgery.

Central Message

CMR imaging provides accurate spatial resolution of regional function of $\mathrm{HM}$ in a swine model that is partially improved at 3 months following $\mathrm{CABG}$.

\section{Perspective}

To understand longitudinal physiologic recovery of hibernating myocardium (HM) following coronary artery bypass grafting, we developed a surgical model of HM in swine that mimics the clinical scenario. Cardiac magnetic resonance imaging (CMR) provides precise regional measurements of impaired function in HM. With revascularization, CMR imaging demonstrates delayed myocardial functional reserve recovery following coronary artery bypass graft surgery at 1 and 3 months.

See Editorial Commentary page 591.
From the ${ }^{\mathrm{a} D e p a r t m e n t}$ of Surgery, University of Minnesota; and ${ }^{\mathrm{b} C a r d i o l o g y}$ Division, Minneapolis VA Medical Center, Minneapolis, Minn.

Supported by VA Merit Review \#I01 BX000760 (to R.F.K.) from the US Department of Veterans Affairs Biomedical Laboratory Research and Development and National Center for Advancing Translational Sciences of the National Institutes of Health Award No. UL1TR000114. The contents of this work do not represent the views of the US Department of Veterans Affairs of the US Government or the official views of the National Institutes of Health.

Read at the 96th Annual Meeting of The American Association for Thoracic Surgery, May 14-18, 2016, Baltimore, Maryland.

Received for publication June 6, 2016; revisions received Sept 16, 2016; accepted for publication Oct 7, 2016; available ahead of print Dec 7, 2016

Address for reprints: Laura L. Hocum Stone, PhD, Department of Surgery, University of Minnesota, 420 Delaware St SE, Minneapolis, MN 55455 (E-mail: stone337@ umn.edu).

0022-5223/\$36.00

Copyright (c) 2016 by The American Association for Thoracic Surgery

http://dx.doi.org/10.1016/j.jtcvs.2016.10.089
Hibernating myocardium (HM) is characterized by the presence of viable yet dysfunctional myocardium in the presence of reduced regional blood flow. ${ }^{1}$ Despite impaired contractility and metabolic activity at rest, an HM is able to demonstrate functional and metabolic reserve under

Scanning this QR code will take you to a video for the article. To view the AATS 2016 Webcast, see the URL next to the video thumbnail.

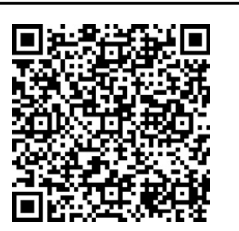



Abbreviations and Acronyms
$\mathrm{CABG}=$ coronary artery bypass graft
$\mathrm{CMR}=$ cardiac magnetic resonance
$\mathrm{HM}=$ hibernating myocardium
LAD $=$ left anterior descending artery
LITA $=$ left internal thoracic artery
PET = positron emission tomography

inotropic stimulation. ${ }^{2} \mathrm{HM}$ is suspected in a majority of patients with coronary artery disease, and encompasses a broad spectrum of disease. The ideal imaging study to identify patients with suspected HM that will recover with revascularization is not yet available. Imaging methods that detect myocardial viability in areas of regional dysfunction include echocardiogram, which assesses wall thinning, contractile reserve, and coronary flow reserve ${ }^{3}$; single-photon emission computed tomography, which assesses residual coronary blood flow and cell membrane integrity to identify viable tissue ${ }^{4}$; positron emission tomography (PET), which measures perfusion and metabolic activity $^{5}$; and cardiac magnetic resonance (CMR) imaging, which is able to measure wall thinning, contractile reserve, perfusion, and tissue scarring. ${ }^{6}$ Of these imaging modalities, CMR demonstrates the highest sensitivity while assessing the most current range of outcome measures. ${ }^{7}$

Although CMR imaging has been used clinically to identify $\mathrm{HM}$, its application in a large animal model has not been previously described. To advance our understanding of the pathophysiology of HM and myocardial recovery, other clinically relevant imaging modalities have been used in large animal models. In swine models, echocardiography has clear utility for viability assessment, most commonly in combination with low-dose dobutamine stress for assessment of regional wall thickening changes. ${ }^{2,8,9}$ However, imaging in swine has an inherent echocardiographic limitation of the acoustic window, making it a less-than-ideal modality for regional left ventricular wall function analysis. PET imaging in swine models of HM has successfully been used to study blood flow, metabolism, and sympathetic neuronal function but lacks the ability to demonstrate anatomy and regional function.,10 Cardiac-gated computed tomography can show late contrast enhancement of the myocardium similar to that seen on CMR imaging, but in swine models it has had greater use as a noninvasive method of defining coronary anatomy. ${ }^{9}$ In clinical studies, segmented cine CMR imaging in preclinical settings has been shown to be an accurate and reproducible modality for the measurement of regional left ventricle function, due to its superior resolution and operator independence. ${ }^{11-13}$
Although used in models of myocardial infarction, CMR imaging in a swine model of hibernation with or without revascularization has not been previously characterized.

In this study, we used our established swine model of HM bypassed with left internal thoracic artery (LITA) to left anterior descending artery (LAD) that mimics the clinical scenario. Swine provide a good model of heart disease over other large animals because they do not have epicardial bridging collaterals. This allows stenosis of the LAD alone to result in regional ischemia. ${ }^{14}$ Because recovery of HM following coronary artery bypass graft (CABG) surgery can result in improved outcome after 3 months in patients, we used a similar time frame to provide optimal myocardial recovery because the degree of functional improvement varies among individuals. ${ }^{15}$ CMR imaging is a sensitive, yet noninvasive method of measuring cardiac function as well as anatomy in this animal model of regional HM that was revascularized surgically. Our previous work ${ }^{9}$ demonstrated by echocardiography that these animals have partial but incomplete recovery 1 month following bypass. In this study, we use CMRenhanced spatial resolution to determine whether functional recovery is complete 3 months following CABG surgery. We hypothesized that recovery would remain incomplete at 3 months post-CABG.

\section{METHODS}

\section{Animal Use}

All animal studies were approved by the Institutional Animal Care and Use Committees of the Minneapolis VA Medical Center and the University of Minnesota and conform to current National Institutes of Health guidelines for the use and care of laboratory animals.

\section{Swine Model of Myocardial Ischemia and Revascularization}

Chronic hibernation model (8- to 10-kg pigs). Animals were sedated with telazol ( $4 \mathrm{mg} / \mathrm{kg}$ administered intramuscularly) and

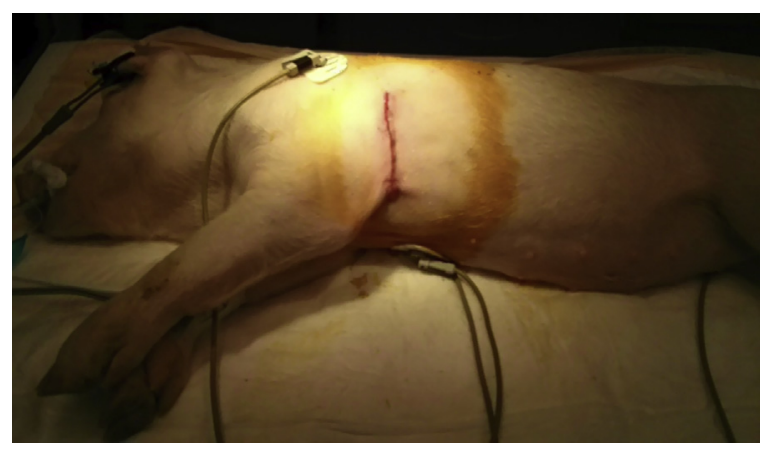

VIDEO 1. This video outlines the surgical techniques employed to establish a swine model of hibernating myocardium, followed by a left internal mammary artery- left anterior descending artery-coronary artery bypass graft procedure and the corresponding cardiac magnetic resonance imaging analysis. Video available at: http://www.jtcvsonline.org/article/S00225223(16)31498-2/addons. 


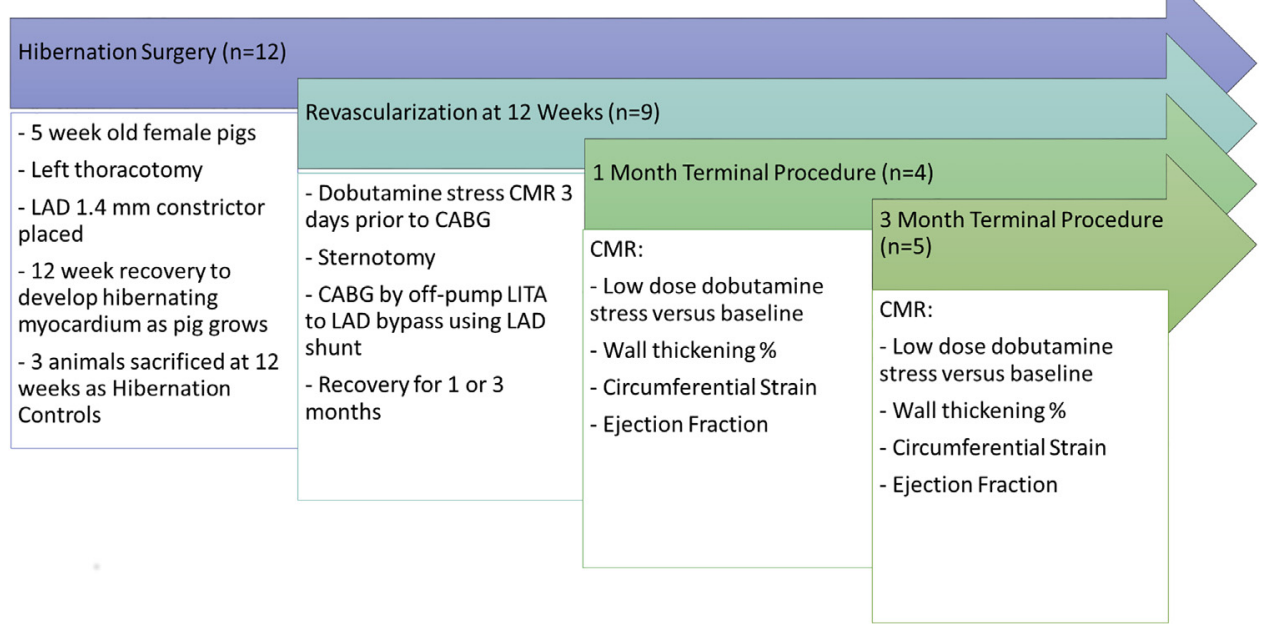

FIGURE 1. Study design creating swine model of hibernating myocardium followed by revascularization. Recovery was assessed at 1 or 3 months using cardiac magnetic resonance $(C M R)$ imaging. $L A D$, Left anterior descending artery; CABG, coronary artery bypass graft; LITA, left internal thoracic artery.

xylazine $(2 \mathrm{mg} / \mathrm{kg}$ administered intramuscularly), intubated, and anesthetized with isoflurane $(2 \%)$. Using a left thoracotomy approach, the LAD had a plastic $C$-shaped constrictor with an internal diameter of $1.5 \mathrm{~mm}$ placed proximal to the first diagonal without occluding the vessel and secured with nonabsorbable sutures. The animal recovered for 12 weeks. We documented proximal LAD stenosis by CMR imaging and at necropsy by direct vessel diameter measurement. Myocardial viability was confirmed by normal gadolinium enhancement in all animals. At 12 weeks, animals underwent the second survival operation of revascularization (see Video 1).

Revascularization model (50- to 60-kg pigs). Animals were again sedated, intubated, and anesthetized as described above. Coronary artery revascularization was performed via a midline sternotomy with the LITA pedicle graft dissected free from the chest wall. Lidocaine $(1 \mathrm{mg} /$ $\mathrm{kg})$ and heparin $(200 \mathrm{U} / \mathrm{kg})$ were administered. The LAD distal to the site of stenosis was then exposed. Using an off-pump technique and coronary shunt, an LITA anastomosis to the LAD was performed. A second CMR imaging study was conducted either 1 or 3 months after revascularization.

Terminal procedure (70- to $80-\mathrm{kg}$ pigs; 120 - to $130-\mathrm{kg}$ pigs). At either 1 or 3 months after revascularization, a terminal surgery was performed (Figure 1). LAD vessel diameter was measured by coronary dilators pre- and postconstrictor and at the anastomotic site with the LITA. LITA diameter was measured in a similar fashion. Graft patency and tissue viability were visually confirmed at this time.

\section{Animal Group Assignments}

Animals were randomly assigned to 1 of 3 groups: hibernation only $(\mathrm{n}=3)$, hibernation with bypass and 1 month recovery $(\mathrm{n}=4)$, and hibernation with bypass and 3 months of recovery $(n=5)$. All animals received CMR imaging before termination, and both bypass groups received CMR imaging before bypass surgery. A 2-tailed post hoc power analysis for the primary end point revealed a power of 1 , based on $\alpha=0.05$, Cohen's $d=2.97$, and a critical $t$ of 2.3 using G*Power (Heinrich-Heine University of Dusseldorf, Dusseldorf, Germany). ${ }^{16}$

\section{CMR Imaging}

CMR imaging was performed on a 1.5-Tesla clinical scanner (Siemens Aera; Siemens Medical Systems, Islen, NJ) with a phased-array 4- channel surface coil and echocardiogram gating. ${ }^{17}$ Animals were intubated and anesthetized with $2 \%$ inhaled isoflurane and positioned in a supine position within the scanner. Left ventricular global function was evaluated and quantified via short-axis cine images with preset slice thickness and spacing using the software package CIMRA (CSON Medical, Minneapolis, Minn). Grid-tagged short-axis cine images, midventricular slices immediately proximal to the papillary muscles, were acquired for the measurement of maximum regional circumferential shortening. Strain measurements were calculated using HARP (Diagnosoft, Durham, NC), as previously described. ${ }^{17,18}$ The above protocol was acquired during rest and repeated during dobutamine challenges of $5 \mu \mathrm{g} / \mathrm{kg} / \mathrm{min}$. Viability imaging was performed following completion of the stress imaging to assess the presence of myocardial infarction using delayed contrast enhancement. Delayed enhancement images were acquired 10 to 15 minutes following the injection of $0.15 \mathrm{mmol} / \mathrm{kg}$ gadolinium-based contrast agent gadobenate dimeglumine (Bracco Diagnostics Inc, Monroe Township, NJ). Remote segments of the left ventricle were defined as the lateral segments of the LV wall opposite the regions that are supplied by the LAD.

\section{Statistical Analysis}

The primary end point measure in this study was regional wall thickening, and secondary end point measures were ejection fraction and circumferential strain. Data are expressed as boxplots depicting the interquartile ranges, the mean, and individual raw data points for each measure. Outliers were determined using the modified Thompson's Tau test and were removed from analysis. Wall thickening measures of remote and LAD regions were compared with a paired 2-sided $t$ test. Analysis of variance was used to determine differences in circumferential strain between groups. Some data points were removed from the strain analysis due to poor image quality. Tukey's post hoc test was used to determine significance between groups in instances of multiple comparisons. Statistics were calculated using GraphPad PRISM software (GraphPad Software, Inc, La Jolla, Calif).

\section{RESULTS}

\section{Coronary Anatomy}

CMR imaging (Figure 2) confirms the presence of stenosis of the LAD 12 weeks following constrictor placement, 


\section{A LAD Stenosis}

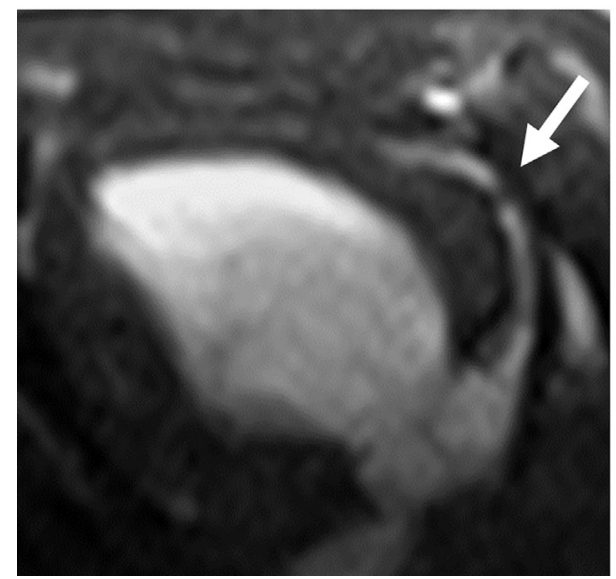

B LITA-LAD

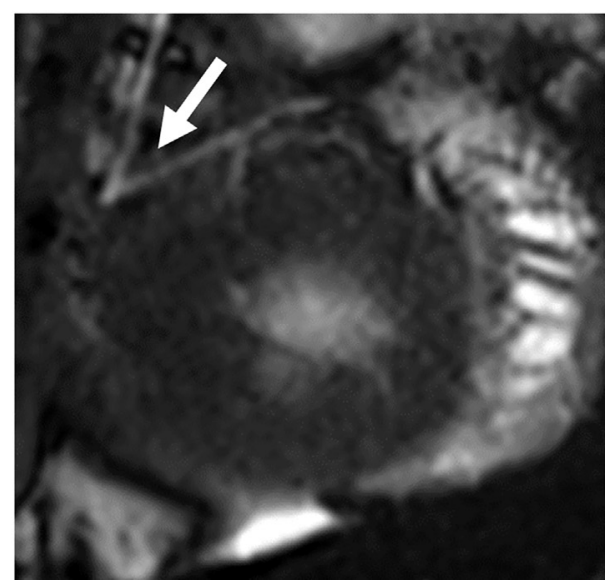

FIGURE 2. Cardiac magnetic resonance images of cardiac anatomy. A, Left anterior descending artery stenosis is present at 12 weeks following constrictor placement. B, Left internal thoracic artery graft is patent 3 months following coronary artery bypass graft surgery.

and confirms that no resulting infarct is present. At 1 and 3 months following CABG, CMR imaging allows for the confirmation of the patency of the LITA graft before the animals were put to death.

\section{Analysis of Regional Myocardial Function}

CMR imaging analysis of regional function revealed a significant decrease in percent wall thickening in hibernating regions at rest $(37.18 \pm 6.57)$ compared with the control remote region $(56.13 \pm 8.06)$ of the left ventricle $(P=.005)$ (Figure 3). Wall thickening at rest showed improvement 1 month and 3 months following revascularization, but remained impaired in the hibernating regions $(31.89 \pm 2.25)$ compared with remote regions
(72.98 \pm 6.41$)$ at 3 months following bypass $(P=.027)$. Under $5 \mu \mathrm{g} / \mathrm{kg} / \mathrm{min}$ dobutamine infusion, wall thickening was significantly impaired in hibernating regions $(46.02 \pm 6.2)$ compared with remote $(91.81 \pm 9.38$; $P=.001)$. Under dobutamine infusion, hibernating regions remained significantly impaired $(54.99 \pm 4.25)$ compared with remote regions $(136.5 \pm 14.84) 3$ months following bypass $(P=.012)$.

\section{CMR Imaging Analysis of Circumferential Strain}

Regional circumferential strain is presented as a ratio of LAD to remote as a method of normalization that controls for differences in hemodynamic parameters, anesthesia, and body size at the time of measurement. At
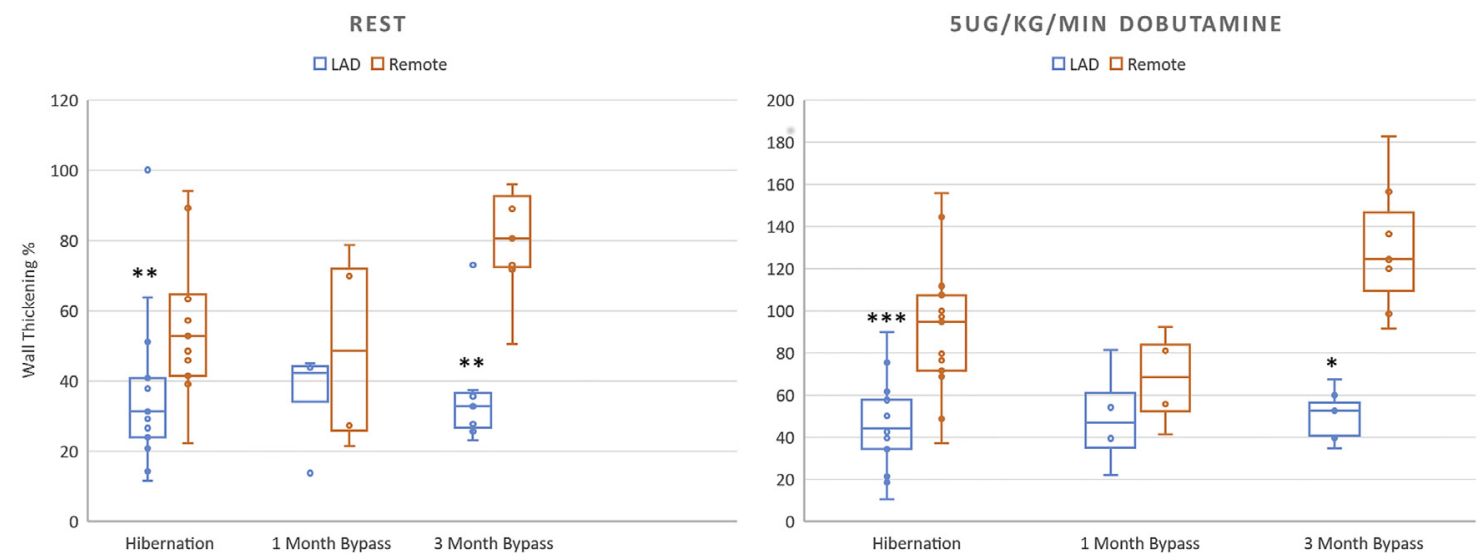

FIGURE 3. Measurement of wall thickening (\%) by cardiac magnetic resonance shows impairment of regional function in hibernating regions of the left ventricle. Wall thickening $(\%)$ is significantly decreased in hibernating animals both at rest and under dobutamine infusion compared with remote regions. Animals undergoing bypass showed improvement in wall thickening both at rest and under dobutamine infusion. $L A D$, Left anterior descending. $* P<.05$. $* * P<.01 . * * * P<.001$. 


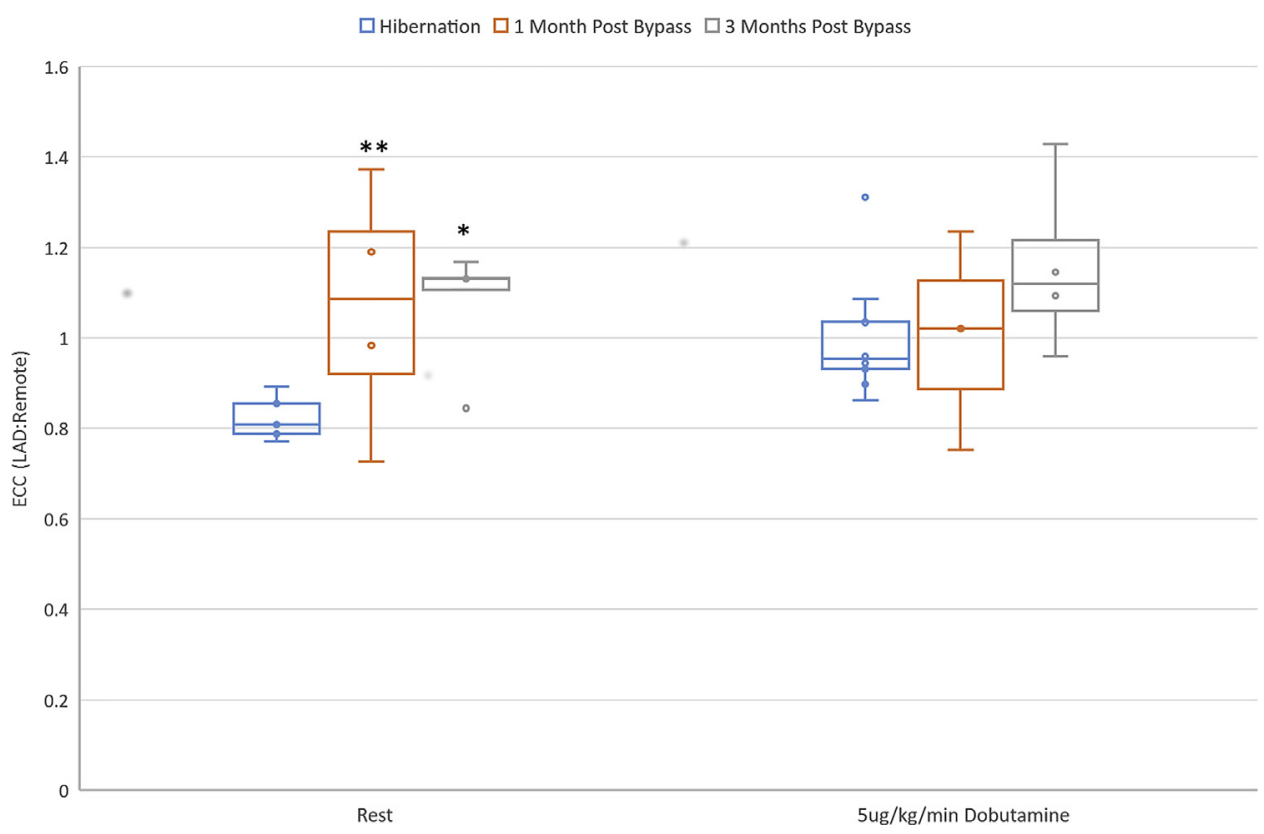

FIGURE 4. Circumferential strain is significantly impaired in hibernating animals, and shows evidence of contractile reserve under dobutamine infusion. Circumferential strain improves at rest, both 1 month $(P<.001)$ and 3 months $(P<.05)$ following coronary artery bypass graft surgery. Strain values are presented as a ratio of the left anterior descending artery $(L A D)$ region compared with the remote region of the left ventricle. ECC, Circumferential strain.

rest, circumferential strain in hibernating animals $(0.823 \pm 0.022)$ is significantly lower than animals 1 month $(1.182 \pm 0.112 ; P=.007)$ and 3 months $(1.076 \pm 0.059$; $P=.023$ ) following bypass surgery (Figure 4 ). The increase in circumferential strain $(0.958 \pm 0.025)$ in hibernating animals during stimulation with $5 \mu \mathrm{g} / \mathrm{kg} / \mathrm{min}$ dobutamine demonstrates the presence of contractile reserve. Under dobutamine infusion, circumferential strain at 1 $(1.002 \pm 0.14)$ and 3 months $(1.066 \pm 0.055)$ following revascularization also shows contractile reserve. High-dose

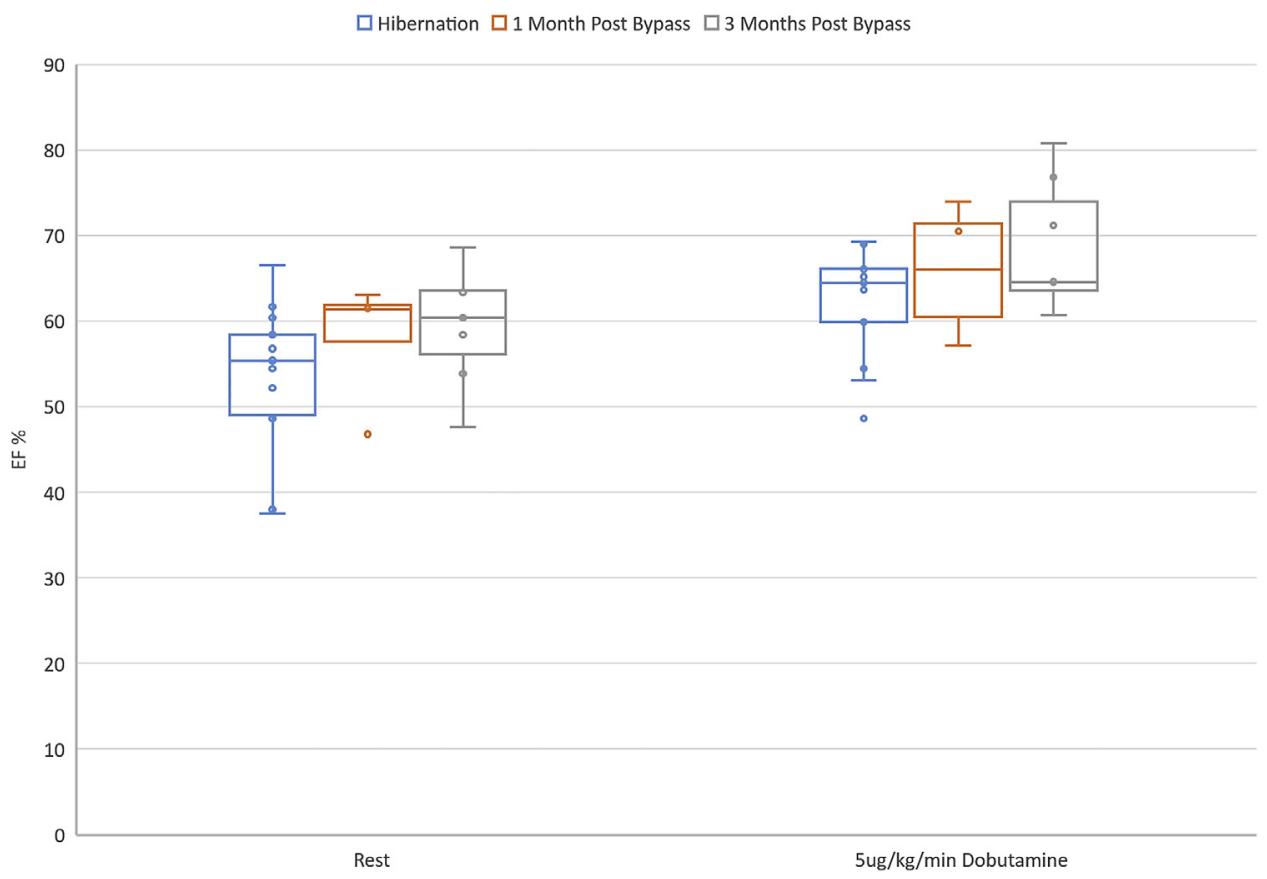

FIGURE 5. Ejection fraction $(E F)(\%)$ as measured by cardiac magnetic resonance imaging does not show an impairment of global cardiac function despite regional hibernating myocardium at rest or under dobutamine infusion. 
infusion of dobutamine was not done due to the inability to achieve quality images for analysis at extremely high heart rates.

\section{CMR Imaging Analysis of Global Function}

Although CMR imaging confirmed the presence of single-vessel stenosis, measurements of global cardiac function by ejection fraction shows no impairment in animals with $\mathrm{HM}(53.57 \% \pm 2.37 \%)$ (Figure 5). This measure of global function remains constant 1 month $(58.18 \% \pm 3.81 \%)$ and 3 months $(59.43 \% \pm 2.89 \%)$ following revascularization and confirmation of patent LITA graft.

\section{DISCUSSION}

Our study shows that our swine model of HM accurately mimics the clinical experience of human patients with single-vessel disease and preserved left ventricular function. Before revascularization, animals with single-vessel HM exhibit minimal impairment in global function as measured by ejection fraction, but show significant reduction in regional wall thickening. The high sensitivity and resolution of CMR imaging, as well as its ability to quantify several measures of function, anatomy, and viability, makes it an ideal tool for the diagnosis of HM and the longitudinal assessment of recovery following CABG. Following revascularization, CMR imaging done at 1 or 3 months of recovery demonstrates preserved viability and graft patency but persistent regional dysfunction, as noted by estimates of contractile reserve with low-dose dobutamine stress testing. The specificity of CMR imaging at defining myocardial regions still at risk for dysfunction despite successful revascularization might be an important tool to assist in targeting individuals who might benefit from additional adjunctive therapies or interventions.

This complex model of revascularization of HM reflects the clinical difficulty of managing patients with viable but chronically ischemic, dysfunctional myocardium. Patients with $\mathrm{HM}$ are frequently encountered in clinic settings, often with various comorbidities and other structural diseases, ${ }^{19}$ and are at risk for sudden cardiac death. ${ }^{20}$ Revascularization of viable, impaired myocardium is associated with a $79 \%$ reduction in annual death rate. ${ }^{21}$ In fact, we have shown that revascularization of patients with viable HM, as defined by PET imaging, is associated with a greater degree of improvement in LV ejection fraction at 6 weeks with CABG. ${ }^{22}$ In several trials of patients with advanced coronary artery disease, CMR imaging has been shown to be an accurate method for determining HM with myocardial viability as determined by the recovery of regional myocardial function after coronary bypass surgery. Selvanayagam and colleagues ${ }^{23}$ delineated the importance of identifying hibernation versus infarction (hyperenhancement) in their study of 52 patients who underwent coronary revascularization. In their study, CMR imaging demonstrated improved regional function in $82 \%$ of segments without preoperative hyperenhancement, in $64 \%$ of segments with $1 \%$ to $25 \%$ hyperenhancement, and $37 \%$ of segments with $26 \%$ to $50 \%$ hyperenhancement. ${ }^{23}$ This confirms that the ability of CMR imaging to predict restoration of myocardial function after revascularization is actually best in segments with the most severe initial dysfunction. The high spatial resolution of magnetic resonance imaging allows for a more accurate determination of the transmural extent of viability as opposed to a binary classification of a myocardial wall segment as either viable or not, as determined by nuclear medicine techniques and echocardiography. ${ }^{24}$ This distinction is critical because it has been repeatedly shown that revascularization of patients with viable myocardium leads to significant improvement in clinical outcome. When only segments with severe preoperative dysfunction were considered, the positive and negative predictive values of CMR imaging for the recovery of function were $81 \%$ and $72 \%$, respectively. ${ }^{23}$ The assessment of regional dysfunction by circumferential strain and regional wall thickening confirms the specificity of HM territory and recovery. Additionally, CMR imaging use in conjunction with low-dose dobutamine infusion has been shown to be a safe and accurate predictor of functional recovery in patients with $\mathrm{CAD}$, and is superior to delayed enhancement imaging in its ability to identify false-negative patients. ${ }^{25,26}$ These capabilities of CMR imaging provide a comprehensive approach for diagnosis of $\mathrm{HM}$ and potential for recovery.

Our use of CMR imaging to compare the response of $\mathrm{HM}$ over time following CABG in a swine model is novel. It provides ideal spatial resolution of the anterior wall within the distribution of the LAD during recovery of regional myocardial function. In hibernating animals, circumferential strain is impaired at baseline, but there is evidence of contractile reserve under inotropic stimulation with a low dose of dobutamine. Presence of contractile reserve is among the most specific indicators of myocardial viability, ${ }^{27}$ and the presence of such viability is a predictor of the potential benefit of bypass surgery when present. In our study, CMR imaging demonstrated that in animals that underwent $C A B G$ surgery, regional function improved at baseline. However, complete recovery evident during high-dose dobutamine stress is likely impaired, even at 3 months, based on our previous studies that showed electron transport proteins remained depressed. ${ }^{8}$ Although the trend is toward continuing improvement at 3 months compared with 1 month of recovery, regional dysfunction despite viability suggests that some of the adaptations with HM may require additional interventions beyond revascularization. 
The incomplete recovery of HM with revascularization reflects clinical experience (Surgical Treatment for Ischemic Heart Failure [STICH] trial). A role for adjuvant therapy with revascularization may be more relevant to hibernation than infarction because the presence of viable tissue provides a more appropriate environment for full recovery. Our previous studies in swine models of HM have shown that at 4 weeks post-CABG surgery, regional function was improved but remained submaximal, despite the presence of a patent graft and improved regional blood flow. Further understanding of the mechanisms underlying $\mathrm{HM}$ is needed, specifically in terms of the time course of recovery of metabolic function in hibernating tissue, because revascularization may not be sufficient to fully restore the metabolic and proteomic profile of cardiac tissue impaired by HM. Targeted therapies that enhance HM recovery may be vital to restore bioenergetic and physiologic responses to their normal state.

CMR imaging has become the gold standard for quantitative analysis of global and regional cardiac function. It also provides high-resolution imaging for the identification of scar presence and tissue viability, as well as quantification of regional blood flow and cardiac strain. ${ }^{28}$ This study for the first time provides an analysis of HM and recovery in a swine model using CMR. The finding of persistent regional dysfunction with dobutamine stress despite a normal global ejection fraction highlights the importance of appropriate imaging in this model and confirms that prolonged recovery may still not restore myocardial function to normal despite successful revascularization.

\section{Limitations}

To successfully induce gradual chronic ischemia in swine, it is necessary to implant a constrictor on the LAD of a juvenile pig, because young swine (aged 5 weeks) have LADs that are small enough to place the constrictor around without creating immediate stenosis. We are unable to create this model by beginning with adult pigs, although that would more closely simulate the human clinical experience, due to size limitations of both the constrictor as well as our surgical and magnetic resonance imaging equipment.

This animal model of regional dysfunction with preserved ejection fraction allows us to only analyze the effects of a single territory of chronic ischemia, whereas clinical cases are typically far more complex, and may respond differently to revascularization. Also, although a second operation adds to the complexity of the model, we performed $\mathrm{CABG}$ rather than percutaneous revascularization due to the rigid nature of the constrictor that was placed.

All regional measurements of function in this study compared hibernating regions to a remote internal control area. Although we acknowledge that this region is not entirely normal in terms of function, it is the best available region for normalizing measurements of function to control for differences in animal size, anatomy, hemodynamic parameters, and anesthesia. It is also possible that the remote, nonhibernating region may become hyperfunctional to compensate for the impairment of the hibernating region. Additionally, there is an innate heterogeneity in the regional function of the LV, although these differences are subtle. Finally, although CMR imaging has the ability to quantify perfusion, the results from our large animal study in which we are limited by small sample sizes resulted in high variability that was difficult to interpret.

\section{CONCLUSIONS}

CMR imaging confirms that HM is present in swine 12 weeks following the surgical placement of a constrictor on the proximal LAD. CMR imaging provides accurate quantification of global and regional function, showing impaired wall thickening and circumferential strain in hibernating regions, although global ejection fraction is preserved. With 3 months of recovery following revascularization, CMR imaging provides an accurate, noninvasive method to analyze regional function and confirm coronary and graft anatomy. The swine model reflects clinical experience, allowing a precise and reliable means to further understand the temporal recovery of HM with CABG surgery.

\section{Webcast}

You can watch a Webcast of this AATS meeting presentation by going to: http://webcast.aats.org/2016/Video/ Tuesday/05-17-16_Ballroom_IV_0720_Kelly-800.mp4.

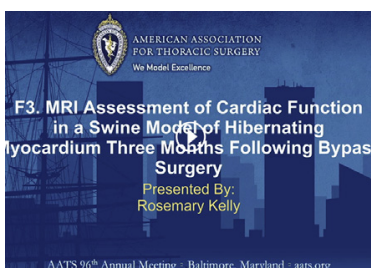

\section{Conflict of Interest Statement}

Authors have nothing to disclose with regard to commercial support.

The authors thank Dr Weihua Guan for providing statistical support.

\section{References}

1. Rahimtoola SH. The hibernating myocardium. Am Heart J. 1989;117:211-21.

2. Fallavollita JA, Logue M, Canty JM Jr. Stability of hibernating myocardium in pigs with a chronic left anterior descending coronary artery stenosis: absence 
of progressive fibrosis in the setting of stable reductions in flow, function and coronary flow reserve. J Am Coll Cardiol. 2001;37:1989-95.

3. Chelliah RK, Hickman M, Kinsey C, Burden L, Senior R. Myocardial contrast echocardiography versus single photon emission computed tomography for assessment of hibernating myocardium in ischemic cardiomyopathy: preliminary qualitative and quantitative results. J Am Soc Echocardiogr. 2010; 23:840-7.

4. Bax JJ, Wijns W, Cornel JH, Visser FC, Boersma E, Fioretti PM. Accuracy of currently available techniques for prediction of functional recovery after revascularization in patients with left ventricular dysfunction due to chronic coronary artery disease: comparison of pooled data. J Am Coll Cardiol. 1997; 30:1451-60.

5. Schinkel AF, Bax JJ, Poldermans D, Elhendy A, Ferrari R, Rahimtoola SH. Hibernating myocardium: diagnosis and patient outcomes. Curr Prob Cardiol. 2007;32:375-410

6. Wellnhofer E, Olariu A, Klein C, Gräfe M, Wahl A, Fleck E, et al. Magnetic resonance low-dose dobutamine test is superior to SCAR quantification for the prediction of functional recovery. Circulation. 2004;109:2172-4.

7. Gurunathan S, Ahmed A, Senior R. The benefits of revascularization in chronic heart failure. Curr Heart Fail Rep. 2015;12:112-9.

8. McFalls EO, Kelly RF, Hu Q, Mansoor A, Lee J, Kuskowski M, et al. The energetic state within hibernating myocardium is normal during dobutamine despite inhibition of ATP-dependent potassium channel opening with glibenclamide. Am J Physiol Heart Circ Physiol. 2007;293:H2945-51.

9. Kelly RF, Cabrera JA, Ziemba EA, Crampton M, Anderson LB, McFalls EO, et al. Continued depression of maximal oxygen consumption and mitochondrial proteomic expression despite successful coronary artery bypass grafting in a swine model of hibernation. J Thorac Cardiovasc Surg. 2011; 141:261-8.

10. McFalls E, Baldwin D, Palmer B, Marx D, Jaimes D, Ward H. Regional glucose uptake within hypoperfused swine myocardium as measured by positron emission tomography. Am J Physiol Heart Circ Physiol. 1997;272: H343-9.

11. Semelka RC, Tomei E, Wagner S, Mayo J, Kondo C, Suzuki J, et al. Normal left ventricular dimensions and function: interstudy reproducibility of measurements with cine MR imaging. Radiology. 1990;174(3 Pt 1):763-8.

12. Barkhausen J, Ruehm SG, Goyen M, Buck T, Laub G, Debatin JF. MR evaluation of ventricular function: true fast imaging with steady-state precession versus fast low-angle shot cine $\mathrm{mr}$ imaging: feasibility study. Radiology. 2001:219:264-9.

13. Grothues F, Smith GC, Moon JC, Bellenger NG, Collins P, Klein HU, et al. Comparison of interstudy reproducibility of cardiovascular magnetic resonance with two-dimensional echocardiography in normal subjects and in patients with heart failure or left ventricular hypertrophy. Am J Cardiol. 2002; 90:29-34.

14. White FC, Carroll SM, Magnet A, Bloor CM. Coronary collateral development in swine after coronary artery occlusion. Circ Res. 1992;71: 1490-500.

15. Shah BN, Khattar RS, Senior R. The hibernating myocardium: current concepts, diagnostic dilemmas, and clinical challenges in the post-STICH era. Eur Heart J. 2013;34:1323-36.

16. Faul F, Erdfelder E, Buchner A, Lang AG. Statistical power analyses using G*Power 3.1: tests for correlation and regression analyses. Behav Res Method. 2009; 41:1149-60.

17. Xiong Q, Ye L, Zhang P, Lepley M, Swingen C, Zhang L, et al. Bioenergetic and functional consequences of cellular therapy activation of endogenous cardiovascular progenitor cells. Circ Res. 2012;111:455-68.

18. Jerosch-Herold M, Swingen C, Seethamraju RT. Myocardial blood flow quantification with MRI by model-independent deconvolution. Med Physics. 2002;29: 886-97.

19. Cooper HA, Braunwald E. Clinical importance of stunned and hibernating myocardium. Coron Artery Dis. 2001;12:387-92.

20. Canty JM Jr, Fallavollita JA. Hibernating myocardium. J Nucl Cardiol. 2005;12: 104-19.

21. Gerber BL, Rousseau MF, Ahn SA, le Polain de Waroux JB, Pouleur AC, Phlips T, et al. Prognostic value of myocardial viability by delayed-enhanced magnetic resonance in patients with coronary artery disease and low ejection fraction: impact of revascularization therapy. J Am Coll Cardiol. 2012;59: 825-35.

22. McFalls EO, Baldwin D, Kuskowski M, Liow J, Chesler E, Ward HB. Utility of positron emission tomography in predicting improved left ventricular ejection fraction after coronary artery bypass grafting among patients with ischemic cardiomyopathy. Cardiology. 2000;93:105-12.

23. Selvanayagam JB, Kardos A, Francis JM, Wiesmann F, Petersen SE, Taggart DP et al. Value of delayed-enhancement cardiovascular magnetic resonance imaging in predicting myocardial viability after surgical revascularization. Circulation. 2004; 110:1535-41.

24. Kim RJ, Wu E, Rafael A, Chen EL, Parker MA, Simonetti O, et al. The use of contrast-enhanced magnetic resonance imaging to identify reversible myocardial dysfunction. N Engl J Med. 2000;343:1445-53.

25. Kelle S, Roes SD, Klein C, Kokocinski T, de Roos A, Fleck E, et al. Prognostic value of myocardial infarct size and contractile reserve using magnetic resonance imaging. J Am Coll Cardiol. 2009;54:1770-7.

26. Kim RJ, Manning WJ. Viability assessment by delayed enhancement cardiovascular magnetic resonance will low-dose dobutamine dull the shine? Circulation 2004;109:2476-9.

27. Pasquet A, Lauer M, Williams M, Secknus MA, Lytle B, Marwick TH. Prediction of global left ventricular function after bypass surgery in patients with severe lef ventricular dysfunction. Impact of pre-operative myocardial function, perfusion, and metabolism. Eur Heart J. 2000;21:125-36.

28. Sawlani RN, Collins JD. Cardiac MRI and ischemic heart disease: role in diag nosis and risk stratification. Curr Atheroscler Rep. 2016;18:23.

Key Words: hibernating myocardium, cardiac MRI, ischemia

\section{Discussion}

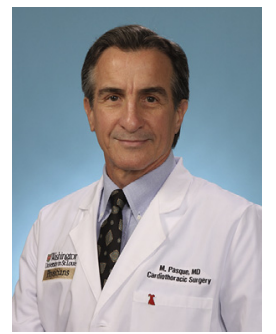

Dr Michael K. Pasque (St Louis, Mo). As I was listening to your presentation, I was reminded of why I am such a big fan of your lab and the work you have done on hibernating myocardium. I love great science and this is great science. You have taken the time to truly validate each component of your model; you have documented the impaired function of the hibernating region; you have documented contractile reserve; you have also made sure that you had coronary stenoses and that you had patent grafts; and most importantly, you documented that there was no myocardial infarction in these hibernating segments. I think that is particularly important.

Hibernating myocardium is foundational to what we do when we operate on coronary bypass patients, and it is especially important to recover these regions of hibernation if we are to optimize outcomes. The measurement of regional contractile function is obviously critical to your study. Most importantly, when you measure regional contractile function, you have to define normal. You have to define a baseline against which you can compare regional contractile function, because everything that you have just discussed is based on that definition, especially regarding whether contractile function returns to where it was at the very beginning before hibernation occurred.

I know you have given a lot of thought to this. There are many different ways to establish a normal baseline and each has its Achilles' heel. We use a normal strain database, whereas some labs look at the regional contractile function before hibernation. Can you give us your thoughts on this? 


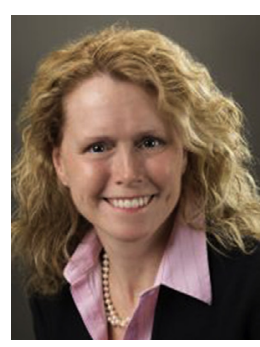

Dr Rosemary F. Kelly. That is an excellent question because we believe the remote region is not completely normal in these animals. In fact, Dr John Canty's group from Buffalo has shown in a similar model that the adaptations remote from the left anterior descending artery undergo several changes at both the functional and molecular levels. There appears to also be a difference in vascular reactivity. However, the remote serves as reasonable control given that each animal has gone through similar stress conditions of 2 operations and prolonged recovery that influences the myocardial regions in a similar fashion. What we are currently underway doing is taking animals of normal weight and size and doing magnetic resonance imaging without any intervention. We have ongoing studies to decipher normal tissue that has not had chronic stenosis, to determine the response in the anterior and septal walls, relative to the inferior and inferolateral walls, which we determine as remote from the left anterior descending artery region. In that way, we can learn more about contractile reserve in tissue that has not been instrumented.

Dr Pasque. Despite the fact that I have spent more than 2 decades trying to convince everyone that the regional contractile impairment associated with valvular cardiomyopathy, especially in aortic insufficiency, almost never returns to normal, I must admit that I was totally blindsided when I read your article. I had never really considered the possibility that truly hibernating myocardium wouldn't return to normal when revascularized. When ejection fractions do not get back to normal clinically we can always rationalize that there is infarction-partial infarction or subendocardial infarction. But I never really imagined that isolated chronic ischemia-without infarction-paralleled what we see in the valvular cardiomyopathy and that in fact it might never get back to baseline.

I am really excited about where you are going with your validated model and this line of research. I think you have the perfect model to see whether it is indeed that, like valvular cardiomyopathy, the duration and the degree of the injury directly correlates with ultimate recovery of contractile function.

I know you have done foundational work on mitochondrial bioenergetics in hibernating myocardium and that there are many different adjunctive therapies that you might use to look at speeding up or completing the recovery of regional contractile function. You have a great model and several potential avenues of research, and it may be time for a little open field running for your lab. Can you give us an idea about where you are headed?

Dr Kelly. Thank you very much for your kind comments. We are in the midst of looking at the metabolic and the bioenergetic alterations in the 3 -month model. We thought the hibernating myocardium should come back to normal by 3 months. The finding that recovery appears to plateau makes us believe that our proteomic studies are going to show a persistent abnormal recovery.

For that reason, we are pursuing other interventions, including utility of antioxidants and possibly cell-based therapies, to promote earlier mitochondrial biogenesis via PGC1 $\alpha$-dependent pathways. Those studies are ongoing and the concepts are still being explored.

Dr Pasque. I think you have the premier cardiac surgical model of hibernating myocardium, and I cannot wait to see what you bring us next year.

Dr Kelly. Thank you.

Readers who found these articles interesting may also like to read the following papers found in recent and future issues of our sister publications, Seminars in Thoracic and Cardiovascular Surgery and Operative Techniques in Thoracic and Cardiovascular Surgery!

\section{Acquired: Coronary Artery Disease}

Original Submission: Open Aortic Arch Reconstruction After Coronary Artery Bypass Surgery: Worth the Effort? Eduard Quintana Semin Thoracic Surg 2016; 28:26-35.

Editorial Commentary: Risky Business or Acceptable Risk? Open Arch Repair After Coronary Artery Bypass Surgery. Joseph S. Coselli. Semin Thoracic Surg 2016; 28:36-37.

Original Submissions: Micromorphology of Skeletonized and Pedicled Internal Thoracic and Radial Arteries. Sergey Mamchur. Semin Thoracic Surg 2015; 27:115-120.

Editorial Commentary: Harvesting Arterial Grafts: Barebones or More. Faisal G. Bakaeen. Semin Thoracic Surg 2015; 27:121-122. 